\title{
Measurements of the Transient Development of a Ceiling Layer
}

\author{
STEVE PALM and E. E. ZUKOSKI \\ California Institute of Technology \\ Pasadena, California 91125
}

\begin{abstract}
The results of a study of the transient production of $\mathrm{CO}$ and other species in a ceiling layer during the development of the layer are presented. The aim of this work is to produce a set of data that can be used as a check on models for species production, particularly of carbon monoxide production, in strongly vitiated ceiling layers under transient conditions.
\end{abstract}

Key words: Transient, diffusion flame, species production, carbon monoxide.

\section{INTRODUCTION}

The majority of fire related-deaths are due to the inhalation of carbon monoxide, a combustion product produced in deadly concentrations in fires burning in products formed by high-equivalence ratio fires. As a result, studying the production and concentration evolution of $\mathrm{CO}$ and other product species in confined fires is important if we are to gain insight concerning ways to reduce fatalities in these situations. Past studies have been largely restricted to steady-state situations which have not led to viable models for the production processes. The aim of the experiments reported here is to produce a welldocumented set of data that describes the transient development of the ceiling layer in a hood that can be used to check computer-based models for the production of carbon monoxide in the ceiling layers formed by real fires.

\section{EXPERIMENTAL APPARATUS AND APPROACH}

Consider a room in which a fire enupts such as that shown in the sketch in Figure 1. At first a plume of hot, buoyant combustion products will impinge on the ceiling. For some room configurations, the ceiling jet formed by the impingement process will fan out over the ceiling until it reaches the walls of the room where it will turn downward until the buoyant forces finally fold it back on itself. Finally, as this layer becomes thicker, the velocities near the interface will become small and a well-stratified two-layer environment will be formed in which the lower layer contains fresh, cool air, and the upper layer contains hot, vitiated gas. If the room contains an open window or door, vitiated gas will be vented as the interface passes the level of the opening, see second sketch, and when the rate of heat release in the flame is relatively constant, the interface will reach a quasi-static height as suggested in the third sketch in Figure 1. If the interface is near the base of the fire, a high-equivalence ratio ceiling layer may be formed that is rich in carbon monoxide. 
To model this flow experimentally, an open-bottomed hood is used to model the upper part of the room illustrated in Figure 1. The hood, shown in the sketch of Figure 2, was constructed from mild steel, with a wall thickness of $0.5 \mathrm{~mm}$, and has a height $\mathrm{H}$ of 1.22 meters, and a width and depth W of 1.83 meters. A $19 \mathrm{~cm}$ diameter natural gas burner is placed $11 \mathrm{~cm}$ below the hood to model the fire. Sufficient air is entrained by the fire plume, as the steady state is approached, to sustain a flame inside the hood even when the oxygen concentration within the hood is nearly zero. Studies of the equilibrium conditions of such experiments have been carried out by S. J.Toner [1] and J. H. Morehart [2 ], and described in Morehart et al [3]. However, to more fully understand the chemistry involved and to be able to model the production of $\mathrm{CO}$ and other chemical species in such an environment, we need to obtain transient results.

Given the limitations of our instrumentation, we can only obtain data from one position within the hood during an experiment. The probe is then moved to a second location and the experiment is repeated while holding all other boundary conditions as nearly constant as possible. Before each test, the hood is cooled to room temperature and filled with room air. This process is repeated with the same size fire and with the probe located at another position until the required number of locations for the probe have been studied.

The results are then combined to obtain a spatial and time resolved data set including the concentrations of $\mathrm{CO}, \mathrm{CO}_{2}, \mathrm{O}_{2}$ and $\mathrm{CH}_{4}$, and the gas temperature. The $\mathrm{CH}_{4}$ tests were incomplete at the time that this paper was submitted for consideration and, because of space limitations, only the data sets for $\mathrm{O}_{2}$ and $\mathrm{CO}$ are described in this report; a complete set of specie data and a more complete description of the experiment is available in Palm and Zukoski [3]. Comparison of data obtained in three nominally identical experiments has shown that the results are repeatable. Given this conclusion, we believe that data, collected from a number of positions within the hood in separate experiments, can be used to develop an accurate picture of the spatially resolved transient processes within the hood.

The sampling probe that was held about $15 \mathrm{~cm}$ away from the center of a side wall and the probe inlet was located at $6,12,35.5,59,82.5$, and $106 \mathrm{~cm}$ above the lower edge of the hood. Wall temperatures are measured at three locations and a thermocouple, placed within the probe inlet and shielded from the direct radiation from the flame, is used to measure the gas temperature. Real-time analysis of the combustion products is provided by four gas analyzers. Calibration gas samples are used to verify the readings before each test and the time delay associated with each instrument was measured so that the data can be shifted to a common time base. A careful investigation by Morehart [2] showed that chemical reactions within the stainless steel probe, which reached temperatures around $450^{\circ} \mathrm{K}$, were negligible for the conditions studied here. Reactions in a copper probe were not negligible.

The mole fraction reported here was determined from a dry gas sample, and the data reported here have not been corrected for the removal of water vapor. The flame was 
allowed to burn for 1800 seconds but data acquisition is continued for another 900 seconds to get information on the hood's return toward ambient conditions.

\section{EXPERIMENTAL RESULTS}

The time required for the system to reach an equilibrium condition is about 1800 seconds and this long period is a result of the small mass flux, about $14 \mathrm{~g} / \mathrm{s}$, that enters the hood when the interface is located only $11 \mathrm{~cm}$ above the surface of the burner. The time constant $\tau_{t}$ defined as the ratio of the mass of gas within the hood to the mass flux carried into the hood by the fire plume is roughly 300 seconds and we expect that the specie concentrations will be within a percent of their final values after a time corresponding to about $5 \tau_{t}$ or 1,500 seconds. This value agrees roughly with that observed here. A second time scale of interest is the time required for the hood to be filled with vitiated gas, called $\tau_{\mathbf{f}}$. Ignoring the effects of heat transfer and assuming that the interface develops as shown in Figure 1, the filling time based on the calculation developed by Zukoski [4] is about $15 \mathrm{~s}$ for this fire.

A fire size of $67.8 \mathrm{~kW}$ and an interface height $Z_{i}$ of $10 \mathrm{~cm}$ were chosen as a standard for this series of tests and the equilibrium state for these conditions, determined earlier by Morehart, is shown here in Table 1.

Table 1. Parameter Values at Steady State Operating Conditions

- Height of top of hood above burner

- Fuel flow rate: Air entrainment rate: Plume Mass flux:

- Heat release rate, stoichiometric combustion Actual heat release rate: (Actual / Stoichiometric) heat release rates

- Upper layer equivalence ratio at steady state: Flame height in unvitiated air
$1.32 \mathrm{~m}$
$1.36 \mathrm{~g} / \mathrm{sec}$
$10.3 \mathrm{~g} / \mathrm{sec}$
$11.7 \mathrm{~g} / \mathrm{sec}$
$67.7 \mathrm{~kW}$.
$27.7 \mathrm{~kW}$
0.409
2.18
$1.10 \mathrm{~m}$

TRANSIENT MEASUREMENTS Having reassured ourselves that the experimental procedure is viable, the distribution of species and gas temperature within the hood was measured as a function of the time for the six probe heights and the results for $\mathrm{CO}, \mathrm{CO}_{2}$, $\mathrm{O}_{2}$ and temperature are shown in Figures 2 to 5 as a function of height; the time after the start of the experiment is shown on each curve at the top of the figure.

Each parameter measured shows the same general change with time. First, a rapid change is observed during the first 100 to 300 seconds as the air within the box is partially replaced or at least mixed with products of combustion, and then a long relaxation time is required to closely approach the steady state condition. However, in these tests, $\mathrm{CO}$ is 
present before $20 \mathrm{sec}$ even though, based on steady state results, we expected that carbon monoxide production would only start after the oxygen concentration in the upper layer was reduced below about $10 \%$, i.e., at times greater than $80 \mathrm{sec}$. Quenching of the reactions in the fire plume by contact with the cold walls early in the experiments may be the cause for this early onset of $\mathrm{CO}$ production. Data collected in the current experiments, at 1,800 seconds agree well with the steady state data of Morehart.

Comparison of the data, obtained for all of the heights and presented in Figures 2 to 4 , shows that the concentration of each specie is substantially independent of height after at least $100 \mathrm{sec}$ and, thus, that the gas within the hood is roughly homogeneous. Presumably, this degree of homogeneity is the result of rapid mixing between the hood gas and the fire plume. The rapidity of mixing within the hood can be made more rational by estimating the flow which is entrained from the hood into the fire-plume. Based on the flame length and entrainment rates of a fire plume burning in unvitiated air, the mass flow entrained into the fire-plume is about $0.2 \mathrm{~kg} / \mathrm{s}$. The pertinent time constant for producing a homogeneous layer $\tau_{h}$ is based on the entrained mass flux and the mass of gas within the hood and for the present example is less than $20 \mathrm{~s}$. Since $\tau_{t}$, the time constant for the change in concentration within the hood, is roughly 15 times larger, $\tau_{h}$ appears to be small enough to insure a rough homogeneity within the hood.

In contrast, the temperature measurements presented in Figure 5 show that there is a substantial variation of gas temperature with height which persists throughout the duration of the experiment. This gradient is probably associated with heat transfer from the gas to the walls of the hood that accounts for most of the heat released by combustion within the hood.

The departure from a perfect uniformity in the species concentrations is also made evident by examining these plots. We believe that at least part of the variation of properties with height shown here is due to the experimental technique in which the properties at each elevation are measured in a different experiment. With this technique, a slight error in measuring the time or variation in heat release between experiments can cause large variations with height.

Note that gas samples drawn from the lowest probe locations were contaminated with products of combustion within the first 10 seconds of the experiment and that no sharply defined ceiling layer was formed. During the first 100 to 150 seconds, substantial vertical gradients did exist in these concentrations and the gradients decrease markedly after about 150 seconds. In contrast, the magnitude of the $\mathrm{CO}$ concentrations developed more slowly and were more nearly uniform with height. Finally, the temperature profiles indicated contamination of the lowest levels in the hood early in the experiment and developed a self similar variation with height after about 150 seconds.

In summary, the filling process studied here involved the whole volume of the hood from the beginning of the experiment and no well-defined ceiling layer or interface is seen during these experiments. The largest variations with height occur during the first 150 
seconds of the experiments and, after this period, the profiles are roughly independent of height, but do depend on the time. This result indicates that during the first 150 seconds of the experiment that buoyancy does affect the mixing process within the hood but is not strong enough to produce a well-defined ceiling layer.

The near independence of the $\mathrm{CO}$ profiles with height suggests that chemical reactions are confined to the flame region and do not occur within the hood proper since large vertical compositions gradients are not present later. Given the low gas temperatures within the hood, this result agrees with the discussion given in Morehart [2] in which detailed chemical computations performed by Morehart showed that fast reactions leading to chemical equilibrium would only occur with any speed at temperatures above $800 \mathrm{~K}$.

HEAT BALANCE The heat-release rate resulting from complete combustion in air of the fuel flow for these experiments is $67.7 \mathrm{~kW}$. However, at long times, the oxygen within the hood is consumed and the fuel-air ratio increases to a value 2.18 times the stoichiometric value. For this fuel-rich condition, the heat released by combustion will be reduced because insufficient oxygen is present to completely oxidize the fuel.

For example, Toner [1] and Morehart et al[2]) found that the fuel-air ratio for the gas that enters the hood for the experimental conditions used here is 2.17 times the stoichiometric value and that the corresponding heat release rate was about $40 \%$ of that for complete combustion or about $27 \mathrm{~kW}$. This estimate for the heat release rate was based on a thermochemical calculation based on measured values of the mole fractions of the species in the gas within the hood that were obtained with a gas chromatograph.

The transfer of energy out of the hood by convection is a surprisingly small part of the heat release. At $1,600 \mathrm{~s}$, the temperature on the ceiling of the hood is about $430^{\circ} \mathrm{K}$ and that at the bottom edge is $388^{\circ} \mathrm{K}$. The radiant energy flux from the hood walls and the bottom opening, estimated by assuming the emissivity of the surfaces as one and by ignoring the direct loss from the flame through the open bottom, is 22 to $25 \mathrm{~kW}$. The convective energy transfer is estimated to be about $1.6 \mathrm{~kW}$. Combining the convective and radiative losses gives net heat release estimate of about 24 to $27 \mathrm{~kW}$, that is in good agreement with the chemical calculation mentioned above.

STARTING TRANSIENT When a plume in an enclosed space such as a room is suddenly started, the plume rises until it impinges on the ceiling and then spreads out along the ceiling until it reaches the walls. Under some conditions, this spreading current is reflected from the walls and returns toward the axis of the plume without penetrating toward the bottom of the hood. This returning current is reflected from the plume and the filling process proceeds by the serpentine process that leads to the formation of a sharply stratified ceiling layer that gradually grows in thickness as suggested in Figure 1. In this process mixing occurs at the interface between the plume material and the air originally filling the hood. 
However, the filling process is controlled in part by the geometry of the room and the mixing process described above only occurs when the height of the room is equal to or greater than the width. When the height of the room is equal to or smaller than its width, the momentum of the initial ceiling layer is large enough that the initial hot gas layer penetrates far down the side wall before buoyancy forces act to stop its downward motion. This wall layer then mixes with the ambient gas and produces a thick mixed layer that may occupy a large part of the hood. For this process, mixing occurs throughout a large volume of the hood and is not restricted to the interface region.

The transition between these two modes of filling was first discussed by Baines and Turner [5] and, based on results obtained with salt-water modeling in a cylindrical tank, they concluded that the region of complete mixing near the ceiling only depends on the ratio of plume height $H$ to diameter $D,(H / D)$. They found that the depth of the mixed region formed by the plume when it first impinges on the ceiling increased with the ( $H / D$ ) ratio and their results suggest that the lower bound for the interface of the mixed ceiling layer, $Z_{I}$, is given by:

$$
\begin{array}{rlcccc}
H_{t} / D & = & 0.5 & 0.75 & 1.00 & 1.25 \\
Z_{I} / H_{t} & = & 1.0 & 0.5 & 0.3 & 0.2
\end{array}
$$

For example, in the experiments discussed here, the diameter of the room based on its area is about $2.1 \mathrm{~m}$ and based on the minimum width is $1.83 \mathrm{~m}$. Given a depth of $1.32 \mathrm{~m}$, values of $(H / D)$ are about 0.63 and 0.72 . Thus, based on the salt-water modeling alone, we should expect that the initial depth of the mixed ceiling layer will be as low as $0.5 \mathrm{H}$. The magnitude of the depth of this initial mixing process in the current experiments can be assessed by examining the first 150 seconds of the plots of the vertical distribution of temperature and species shown in Figure 3. These data show that the mixing is strong enough to transport buoyant gas to the bottom of the hood within the first 5 seconds after the initiation of combustion. Thus, mixing in this experiment is more extensive than would be expected from the salt-water/water experiments of Baines and Turner.

The primary difference in these two experiments is that, in the current experiments, the initial temperature of the plume gas after impingement is greatly reduced by convective heat transfer to the ceiling and side walls of the hood. When the plume first impinges on the ceiling, the walls are at room temperature and a large fraction of the heat released by the fire is transferred to the walls of the hood. Thus, the density of the plume gas is very nearly that of the surrounding air and buoyancy forces do not impede the downward motion of the recirculating flow. In the Baines and Turner experiment, buoyancy is produced by the concentration of salt and the interaction with the wall has no effect on the buoyancy of the flow.

This phenomena may be of importance in understanding the initial transient produced in room fires when the fire grows very rapidly at first and approximates the step function change in buoyancy used in these experiments. 
REFILLING PHENOMENA The behavior of the system after the termination of the flame was surprising. At the two lower positions for the probe, at 6 and $12 \mathrm{~cm}$ above the bottom of the hood, the oxygen concentration returns to the value in room air within roughly 20 seconds after the flame is stopped and remains at that value. At higher elevations, the return is slower and the oxygen concentration does not reach room conditions in the 800 seconds of this phase of the experiments. Similar changes occur in both $\mathrm{CO}_{2}$ and $\mathrm{CO}$ concentrations. Several mechanisms are possible and we are investigating them now. One mechanism is associated with the presence of natural convection currents on the walls of the hood. The flux of heat through the walls of the hood keeps the temperature of the walls about $100^{\circ} \mathrm{K}$ below the temperature of the gas and this temperature difference will cause downward-flowing natural convection currents on the side walls. When the fuel flow is stopped, the recirculating currents within the hood rapidly die out and the remaining flow is dominated by these natural convection currents that mix with the ambient air at the bottom edge of the hood and some of the oxygen entrained by this mixing process may enter the hood.

A second mechanism involves the surface of the burner that in our experiments is a bed of glass beads. During the flaming period, these beads are heated to temperatures of the order of $600^{\circ} \mathrm{K}$ and, after the flame is extinguished, they are the source of a natural convection plume that can also entrain air and hence feed oxygen into the hood

\section{DISCUSSION AND CONCLUSIONS}

A partial data set for the transient filling of a hood has been obtained in which carbon monoxide, carbon dioxide, and oxygen concentrations, the gas temperature, and the wall temperature at three positions were measured as a function of time at 6 elevations within a hood that had a height-to-width ratio of $2 / 3$.

The development and descent of a well defined ceiling layer was not observed early in the experiment and products of combustion reached the bottom of the hood within the first few seconds of the experiments. We believe that both geometric effects, discussed by Baines and Turner, and the heat loss to the walls, that reduces the buoyancy of the initial plume flow, caused the initial plume flow to mix with all of the hood gas in the first few seconds after the impingement of the plume on the ceiling of the hood and that these processes prevented the formation of a clearly defined ceiling layer. The relative importance of the geometric effects compared with the heat transfer effects can not be assessed with the current results since only one value of the height to width ratio has been studied.

For the conditions examined here, the mixing within the hood is strong enough to prevent significant stratification of the concentration profiles after the first 150 seconds of the experiment. This is the time during which about half of the concentration changes occur. Any chemical reactions which may occur within the hood are not strong enough at the low gas temperatures present in these experiments to produce a substantial stratification of chemical species. 
Concentration of carbon dioxide and oxygen grew rapidly for the first 100 seconds and then much more slowly for the rest of the test. In contrast, the concentration of carbon monoxide grew relatively more slowly and required more than 300 seconds before the growth rate decreased markedly. All three concentrations approached the steady state values reported earlier by Morehart.

These measurements of transient values for the gas temperature and concentrations of three of the major species presented here can be used in the development of codes to predict the production rate of carbon monoxide and for the filling process in room fires.

Mixing with the ambient air at the interface after the termination of combustion can return oxygen to the lower part of the hood within a few minutes. This process appears to be produced by the mixing of the natural convection currents on the side walls of the hood or by the natural convection plume rising above the surface of the hot burner. We believe that his process can have a strong influence on the development of certain types of accidental fires.

\section{ACKNOWLEDGMENTS}

This work has been supported by the Building and Fire Research Laboratory of the National Institute of Science and Technology, the Department of Commerce under the supervision of Dr. L. Cooper.

\section{REFERENCES}

1. Toner, S. J., Entrainment, Chemistry, and Structure of Fire Plumes, Ph.D. Thesis, Calif. Inst. of Tech., Pasadena, Calif., (1986)

2. Morehart, J. H. Ph.D., "Species Production in Fires Burning in Two-Layered and Honogeneously Vitiated Environmernts, "Thesis, Calif. Institute of Tech., Pasadena, Calif. (1991)

3. Morehart, J. H., Zukoski, E. E., and Kubota T. "Characteristics. of Large Diffusion Flames Burningin a Vitiated Atmosphere, Third Int. Symp. of Fire Safety Science p. $575-583,(1991)$

4. Palm, S. and Zukoski, E. E., Measurements of the Transient Development of a CeilingLayer, Jet Prop. Center Rep., Calif. Inst. of Tech., Pasadena, Calif.,(1993)

5. Zukoski, E. E., "Development of a Stratified Ceiling Layer in the Early Stages of a Closed-Room Fire," Fire and Materials, 2, p. 54.,(1978)

6. Baines, W. D. and Turner, J. J. "Turbulent Buoyant Convection From a Source in a Confined Region," J. Fluid Mech., 37, pp. 51-80, (1969) 


\section{FIGURES}
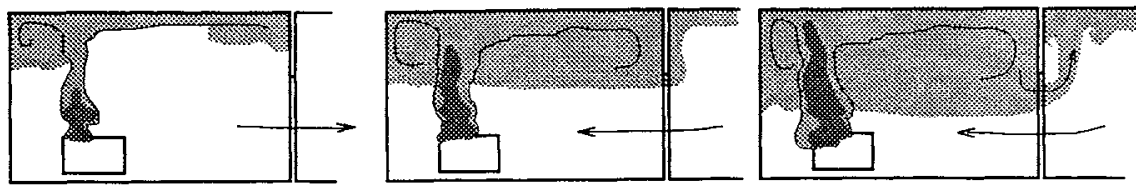

Just after impingement Developing layer

Quasi-static layer

Figure 1. Sketch of the development of a ceiling layer in a room fire.

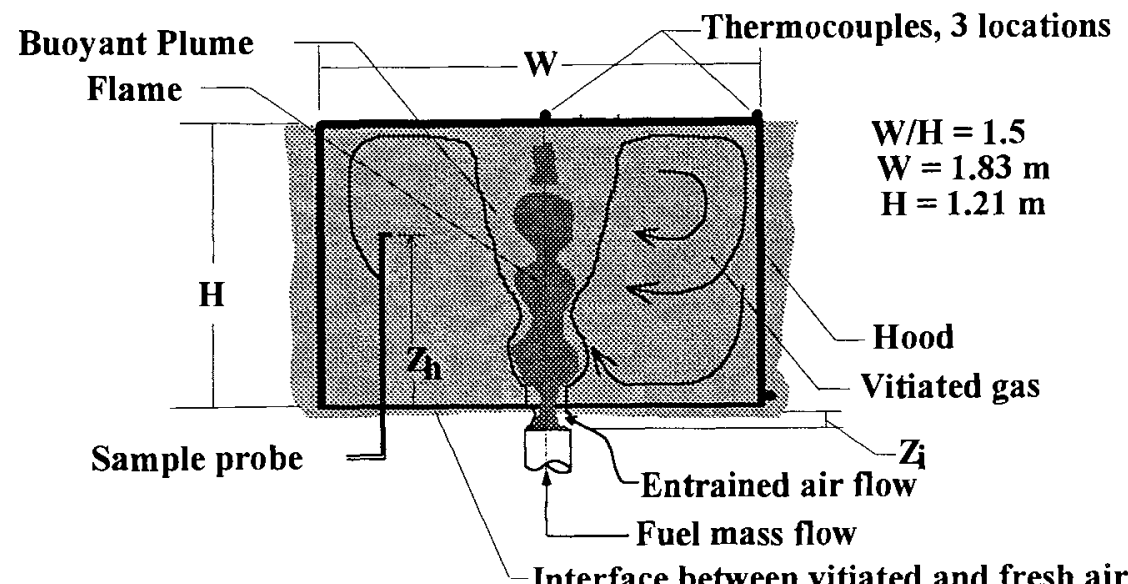

Figure 2. Sketch of hood apparatus 


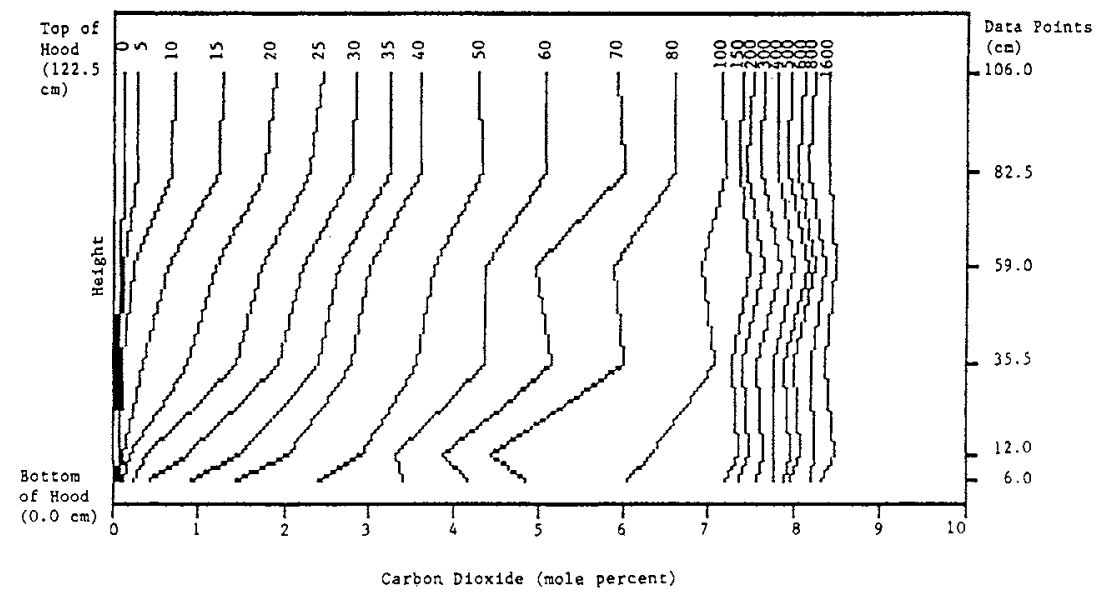

3. Concentration of $\mathrm{CO}_{2}$ as a function of height and time.

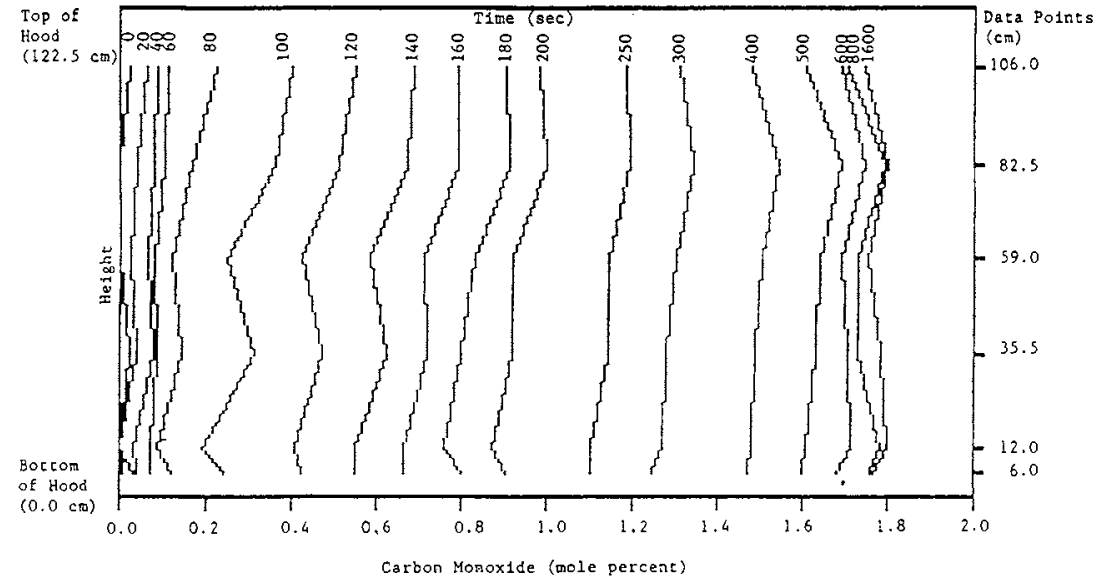

4. Concentration of $\mathrm{O}_{2}$ as a function of height and time. 


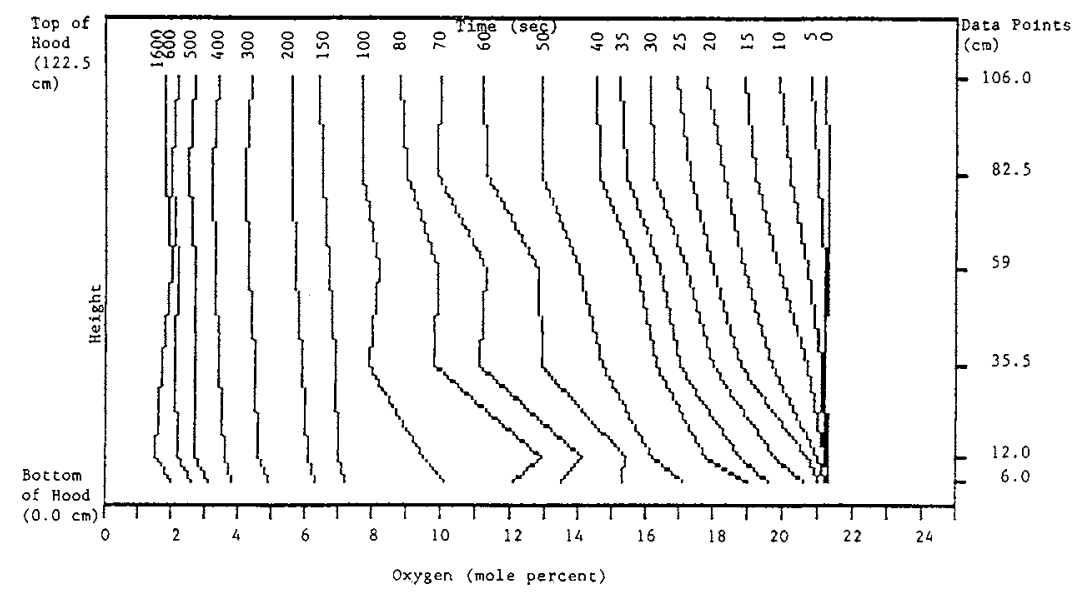

5 Concentration of $\mathrm{CO}$ as a function of height and time.

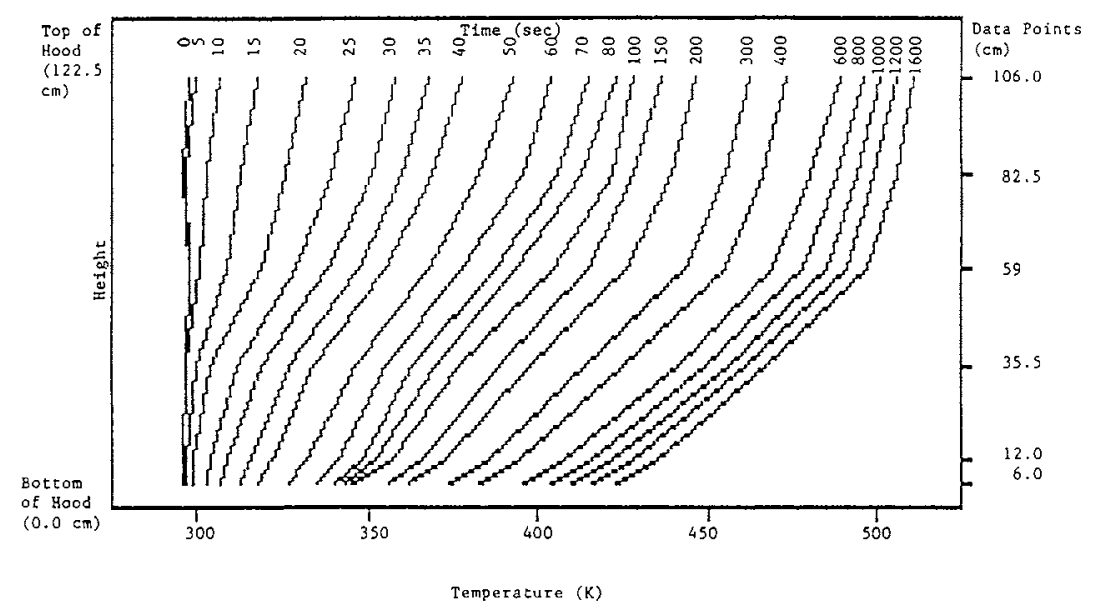

6. Gas temperature as a function of height and time. 
